\title{
Arrival of the 5G Era of Clinical Medicine for Breast Cancer
}

\author{
Nan Zhang, Xiangying Xin, Deqiao Wu, Yan Zhang, Hui Chen, Nannan \\ Feng, Junwei Zhang, Yifan Zhu, Zhenzhong Zhang* and Xia Xu* \\ School of Pharmaceutical Sciences, Zhengzhou University, Zhengzhou, China \\ *Corresponding Author: Zhenzhong Zhang and Xia Xu, School of Pharmaceutical \\ Sciences, Zhengzhou University, Zhengzhou, China.
}

Received: December 23, 2020

Published: January 16, 2021

(C) All rights are reserved by Zhenzhong

Zhang, Xia Xu., et al.

DOI: $10.31080 /$ ASPS.2020.05.0664

\begin{abstract}
Breast cancer is one of the most common malignant tumors among women worldwide. Since the 1970s, the incidence of breast cancer has always been the highest in women with tumors. Its incidence has shown an upward trend. Clinical medication for breast cancer has also been updated owing to social progress and development, particularly in the current mobile data networks. The mobile data network evolved from the $1 \mathrm{G}$ to the $5 \mathrm{G}$ era. Upon consulting literature, combined with the current understanding of the clinical application of breast cancer, the developmental changes and prospects of the clinical application of breast cancer in the $5 \mathrm{G}$ era were summarized. This review focused on the development of breast cancer from the $1 \mathrm{G}$ to $5 \mathrm{G}$ era. Beginning from the advent of the era of efficacy of the $1 \mathrm{G}$ era, represented by the introduction of paclitaxel and doxorubicin, to changes in docetaxel and epirubicin, with the arrival of the transitional era represented by chemical structure. With the advancement of science and technology, we entered the targeted era, represented by nano-preparations such as albumin paclitaxel and liposomal adriamycin. Currently, we are utilizing multi-drug combination therapy.
\end{abstract}

Keywords: Breast Cancer; Clinical Medication; 5G Era

\section{Introduction}

Approximately $17 \%$ of new tumors in women are diagnosed as breast cancer. In 2018, 2.09 million patients were diagnosed with breast cancer [1]. Clinical manifestations of this disease include breast lumps, pain, surface ulcers, nipple discharge, and axillary lymphadenopathy. These manifestations threaten the physical and mental health of female patients [2]. The etiology of breast cancer is complex and its pathogenesis is not well understood. However, some etiological studies have shown that the incidence of breast cancer was closely related to multiple factors. First, having a family history of this disease was a high-risk factor [3]. In 1974, Anderson [4] noted that women with first-degree relatives, with breast cancer, are two to three times more likely to develop the disease.
Second, the occurrence of breast cancer was related to the patient's reproductive factors and the levels of estrogen in the body. This may be affected by early menarche, late menopause, absence of childbearing, withholding breastfeeding, and intake of exogenous hormones [5]. Third, smoking and drinking habits also increased the risk of developing breast cancer [6] because alcohol increases the levels of plasma estrone and estradiol thus altering its hormone balance. Additionally, burning of tobacco produces carcinogens such as nicotine. Recent research shows that BRCA gene mutation was also an important risk factor for the development of breast cancer [7].

The treatment of breast cancer includes surgical treatment, endocrine therapy, chemotherapy and radiotherapy, and combination 
therapy. The treatment methods are continuously being improved, although drug treatment has always been an important treatment method. Medication therapy is currently being improved to optimize patient benefits. Scientists have come to terms with the advancing science and technology. Mobile communication technology, which initially developed from the $1 \mathrm{G}$ era, consisting of only calls, has been inseparable from us. However, currently, we live in the $5 \mathrm{G}$ era, an era with faster, stronger, and smarter communication options. Similarly, as the physician's clinical experience increases, the understanding of breast cancer continues to deepen with more drugs for the treatment of breast cancer being made available. Clinical medications are progressing and evolving to be more efficient, beneficial, and tolerable.

This article compares the development of clinical treatment drugs for breast cancer with the changes from the $1 \mathrm{G}$ to $5 \mathrm{G}$ era of mobile communication technology. Further, it provides an overview of the representative drugs in breast cancer treatment. This article uses doxorubicin and paclitaxel as representative drugs.

$1 \mathrm{G}$ era of clinical medication for breast cancer-paclitaxel, adriamycin

Earlier, drugs for the treatment of breast cancer mainly involved non-anthracyclines and anthracyclines [8]. Prior to adriamycin treatment, non-anthracyclines, cyclophosphamide, methotrexate, and fluorouracil occupied the market for breast cancer treatment. However, none of them were ideal and effective. The emergence of anthracycline chemotherapeutics, represented by doxorubicin, has epoch-making significance in breast cancer chemotherapy and significantly improved the long-term survival rate of breast cancer patients. Doxorubicin was isolated and created by Acramone et al. in Italy in 1969 [9]. It is a cyclic nonspecific drug that kills tumor cells in various growth cycles. Its mechanism of action is mainly to intercalate between DNA base pairs and inhibit DNA topoisomerase II [10]. It was clinically used in the treatment of breast cancer, leukemia, and lymphomas. The main adverse reaction of doxorubicin was irreversible heart damage [11]. This manifested as degeneration and edema of myocardial cells in the early stage, fibrosis, dissolution, or collagen deposition in the late stages, and heart failure or dilated cardiomyopathy in severe cases.

Paclitaxel (PTX) is a fat-soluble diterpene alkaloid compound isolated from the Taxus genus. In 1983, it was used in the clinical treatment of ovarian and breast cancers, as the best natural anticancer drug discovered. This was approved by the US FDA in 1992 [12]. The anti-cancer mechanism of PTX induced and promoted tubulin polymerization, thereby inhibiting tumor cell mitosis and triggering cell apoptosis to achieve anti-tumor effects [13]. The solubility of paclitaxel in water is less than $0.004 \mathrm{mg} / \mathrm{mL}$ [14]. In the clinical application of traditional injections, polyoxyethylene castor oil (Cremophor EL) mixed with absolute ethanol as a solubilizer may lead to severe allergic reactions [15].

Since paclitaxel and doxorubicin are both raw materials, they can be used in a wide range of clinical applications. They were used to treat almost all types of breast cancer patients, including postoperative adjuvant chemotherapy and neoadjuvant chemotherapy. The patients were significantly benefited and underwent treatment for breast cancer. An epoch-making drug in history, owing to its high adverse reactions and poor patient tolerance, had limited application. Therefore, there was a need for drugs with similar efficacy and with less adverse reactions. This demand forces the treatment of breast cancer from the $1 G$ efficacy era into the $2 G$ era-the transition era.

$2 \mathrm{G}$ era of clinical medication for breast cancer-docetaxel, epirubicin

Docetaxel is a taxol derivative synthesized during the process of restructuring paclitaxel. The paclitaxel C-3' benzamide group was substituted with trimethylformyl formamide, and the C-10 acetoxy group was substituted with a hydroxyl group, which is a secondgeneration taxane anti-cancer drug [16]. Docetaxel has the same mechanism of action as paclitaxel. However, the drug concentration and residual time of docetaxel in cells was three times than that of paclitaxel. Further, it had higher anti-tumor activity and bioavailability, wider anti-tumor spectrum, was less toxic, and had fewer side effects. Docetaxel rarely had adverse effects more severe than allergic reactions.

Epirubicin is an isomer of doxorubicin that is also an antibiotic anti-tumor drug. Its mechanism of action is similar to that of adriamycin. Epirubicin has a broad anti-tumor spectrum and high curative effect. It also showed less cardiotoxicity [17]. Clinically, cardiac functions should be routinely evaluated prior to chemotherapy, in order to reduce the cardiotoxicity of doxorubicin and epirubicin. 
During chemotherapy, cardiac troponin and brain natriuretic peptide should be regularly tested, with appropriate adjustment in the dosage or regimen.

The advent of docetaxel and epirubicin overcame the shortcomings of traditional paclitaxel and doxorubicin. The side effects were fewer, and the curative effect improved. Therefore, breast cancer patients who were intolerant to traditional paclitaxel and doxorubicin also benefited. However, docetaxel and epirubicin only changed the chemical structure of the basic chemotherapeutic drugs. Since the mechanism of action was still the same, the use remained limited; passive targeting agents were clinically needed. Therefore, soon the $2 \mathrm{G}$ era was transitioned to the new $3 \mathrm{G}$ era - the targeting era.

3G era of clinical medicine for breast cancer-nano formulations of paclitaxel and adriamycin

The traditional paclitaxel injection solubilizer, polyoxyethylene castor oil, had biological effects and was prone to allergic reactions [15]. Simultaneously, such active agents may form tiny particles in the blood that encapsulates the PTX molecules. Encapsulation affected the drug molecules and prevented them from spreading into the tissues. This was not conducive to the anti-tumor effect ofPTX.

In order to facilitate clinical use and reduce its toxicity and side effects, paclitaxel was relaunched in the market. The development of a new drug delivery system for paclitaxel that does not contain Cremophor EL was targeted as a research topic. New PTX preparations with significant clinical effects, including albumin-bound paclitaxel [18], paclitaxel liposomes [19], and paclitaxel polymer micelles (Genexol-PM), were developed in home country and abroad [20]. They had variable performances in terms of reducing or eliminating allergic reactions, improving tumor targeting, and tolerable doses.

Among them, albumin paclitaxel caused widespread concern in the academic community.

Albumin-bound paclitaxel was a new formulation of paclitaxel, freeze-dried with human albumin as a drug carrier and stabilizer. It was launched in the United States in January 2005 and was available in China in 2009, quickly occupying most of the clinical market as paclitaxel-based treatment of breast cancer [21]. Albumin pa- clitaxel mainly uses the unique targeting transport mechanism of SPARC in the cell membrane to improve the content of intracellular albumin and paclitaxel within tumors [22]. The body concentration of ordinary paclitaxel was generally $135-175 \mathrm{mg} / \mathrm{m} 2$ [23]. However, the

Albumin-bound paclitaxel increased it to $260 \mathrm{mg} / \mathrm{m} 2$ [24]. Patients did not need to undergo pretreatment before taking the drug, which shortened the infusion time and eliminated polyoxyethylene castor oil. Related adverse reactions were well tolerated by patients. Many clinical studies pointed out that albumin PTX treatment of metastatic breast cancer not only improved the overall response rate, but also prolonged the overall survival time. Comprehensive meta-analysis of the literature on the efficacy and safety of albumin and solvent-based PTX on breast cancer showed that the overall remission rate of albumin-PTX for breast cancer was significantly better than that of solvent-based PTX. The grades $3 / 4$ adverse reactions did not have significant differences. It was clinically proven that the application prospect of paclitaxel nanodrug delivery system was good.

Adriamycin cardiotoxicity and other adverse reactions severely limited its clinical application. For several years, medical workers in various countries were committed to adopting a new drug delivery system to reduce drug toxicity, improve efficacy, and improve patient tolerance to drugs. In 1971, Gregoriadis and Rymen were the first to report that liposomes can be used as drug carriers [25]. In the late 1970s, liposomes began to serve as effective carriers for anthracycline anti-tumor drugs. As drug carriers, liposomes have many advantages and characteristics. First, it is nontoxic to the human body. Second, drug release can be effectively controlled under different conditions. Third, encapsulated drugs are not absorbed by blood circulation. Fourth, the size and charge can change and be modified with ligands. Finally, it is absorbed by the reticuloendothelial system (RES) [26]. A meta-analysis of doxorubicin liposomes and traditional doxorubicin preparations, conducted by Chee and Wayne, showed that liposomal doxorubicin chemotherapy significantly reduced the risk of cardiotoxicity and significantly increased the overall remission rate [27]. Adriamycin liposomes combined with other drugs were widely used in the treatment of breast cancer. 
Albumin paclitaxel and liposomal doxorubicin are targeted nano-formulations. Since their inception, they rapidly occupied the market for breast cancer. They have effectively reduced the systemic adverse reactions of raw materials and significantly improved their in vivo efficacy.

Regardless of the dosage form of the drug [28], it was difficult for a single drug to cure refractory and metastatic breast cancer. Therefore, combination therapy became the new treatment trend. Hence, the 4G era, the combination era of clinical breast cancer drugs that contained drugs with different mechanisms of action, was initiated.

4G era of clinical medication for breast cancer - united era

\section{Endocrine drugs}

More than $70 \%$ of breast cancer patients are estrogen or progesterone receptor-positive [29]. Therefore, endocrine therapy drugs are the standard treatment for these patients. The principle of endocrine therapy is to reduce the levels of estrogen in patients or to inhibit the growth of cancer cells. Endocrine drugs include anti-estrogens, SERMs, OFS, aromatase inhibitors (AIs), luteinizing hormone (LH)-releasing hormone analogs, and progesterone drugs. Endocrine therapy has relatively fewer adverse reactions and long-lasting effects, but endocrine therapy drugs increase the occurrence of bone-related adverse events and affect bone metabo$\operatorname{lism}[30]$.

\section{Anti-estrogen drugs}

Reducing the levels of estrogen in the body is beneficial for breast cancer treatment. Tamoxifen citrate (TAM, tamoxifen) [31] was the first preventive drug approved by the FDA to reduce the risk factors of breast cancer women before and after menopause. This served as the standard controlled treatment for the multiple clinical trials of new drugs. TAM was most effective in treating estrogen receptor (ER)-positive patients. It was used in premenopausal women to replace ovariectomy, radiation castration, or metastatic breast cancer. People over years old had better results than premenopausal and younger patients. Common adverse reactions included gastrointestinal reactions, secondary anti-estrogen effects, such as facial flushing, menstrual disorders, amenorrhea, and neuropsychiatric symptoms, such as headache and depression.
Long-term use of large doses caused visual disturbances such as cataract, hair loss, weight gain, and abnormal liver function.

Aromatase inhibitors

AIs are specific and can inhibit or inactivate aromatase, thereby inhibiting estrogen production, and were often used to treat estrogen-dependent breast cancer. Since the ovaries of premenopausal patients is the main organ for estrogen production and AIs cannot completely block the synthesis of estrogen in the ovaries, they were used in postmenopausal breast cancer patients. There are three generations of AIs. The first generation included aminoglutelhimide (Cytadren) [32], the second generation included formestane [33], and the third generation included letrozole [34], anastrozole [35], exemestane [36], and others. The third-generation AI had a clear therapeutic advantage over TAM and thus, became the drug of choice for treating postmenopausal breast cancer patients. Exemestane had the advantages of bone and blood lipid safety [37].

\section{SERM}

SERM is a type of compound that can interact with ERs to produce tissue-specific compounds. Estrogen antagonism is due to competitive binding of SERM to estrogen, preventing the co-regulatory protein that activates or inhibits gene transcription from binding to the surface of AF-2 (the estrogen-constrained gene activation and response functional region) [38]. Commonly used drugs include tamoxifen, toremifene (TOR), raloxifene (RAL), fulvestrant (FVT, faslodex).

\section{Tamoxifen: Same as above}

Toremifene: TOR is a derivative of tamoxifen [39]. Its mechanism of action was similar to that of TAM, and its effect on breast cancer was equal to or higher than that of TAM. This drug is an ER modulator approved by the US FDA for breast cancer after tamoxifen administration. It can replace adjuvant endocrine therapy for early and advanced breast cancer patients who cannot tolerate TAM. Toremifene has a large distribution concentration in the lungs, and hence, it is particularly effective for breast cancer with lung metastasis patients. The adverse reactions of toremifene are mild, and the risk of causing endometrial cancer isonly $33.33-50 \%$ of that of tamoxifen. Although toremifene has certain advantages in endocrine therapy, its curative effect is not significantly better than 
that of TAM. Therefore, it cannot replace the status of TAM as the first-line adjuvant therapy.

- $\quad$ Raloxifene [40]: It has anti-estrogen effects on the uterus and breasts. It is a second-generation SERM drug consumed by postmenopausal women. This drug can be used as a preventive therapy for breast cancer. It was reported that patients who underwent hysterectomy, especially those at high risk, can obtain the greatest benefit and the least harm through the use of RAL or TAM as preventivetreatment. Raloxifene has certain advantages over TAM in terms of reducing the risk ofbreast cancer. Common adverse reactions include facial flushing and leg musclecramps.

- Fulvestrant [41]: Unlike TAM, it only has ER antagonism. Noagonistic effect can effectively reduce the ER and progesterone receptor levels of breast cancer cells, thereby inhibiting the growth of breast cancer cells. It is mainly used in postmenopausal women who received anti-estrogen drugs (such as TAM), but their condition is still getting worse, or the follow-up treatment of third-generation AI resistance.

- Ovarian function inhibitors: This includes ovariectomy, radiotherapy, and drug-induced ovarian function inhibition. The mechanism of action was to acton the hypothalamus through negative feedback to inhibit the production of gonadotropin-releasing hormones (GnRH/LH-RH). Simultaneously, it can also competitively bind to the $\mathrm{GnRH}$ or LH-RH receptors on the pituitary cell membrane to prevent the pituitary gland from producing follicle stimulating hormone (FSH) and LH, thereby reducing the secretion of estrogen from the ovaries [42]. Ovarian function inhibitors overcome the shortcomings of surgery and radiotherapy castration. After stopping the drug, the patient's ovarian functions return to normal. Therefore, it is suitable for breast cancer patients with fertility concerns. The drug has is highly safe while ensuring significant therapeutic effect. Adverse reactions to the drug include flushing, occasional headaches, large mood swings, vaginal dryness, and decreased libido.

- $\quad$ Progesterone drugs: Progesterone can inhibit hypothalamic gonadotropin-releasing hormone, thereby reducing the levels of FSH and LH in the body. At the same time, it induces liver $\alpha$-reductase to increase the degradation rate of estrogen in the body. Thus, it hinders the synthesis of estrogen [43]. Commonly used drugs include medroxyprogesterone and megestrol. The therapeutic effect of this drug is equivalent to that of TAM, but it will produce greater adverse reactions than TAM, showing a progesterone-like reaction, which can effectively treat postmenopausal breast cancer patients.

\section{Molecular targeted therapy drugs}

Molecular targeted therapy [44] is an emerging tumor treatment model. Compared to traditional chemotherapy, it has the advantages of strong specificity, low dosage, good tolerance, and less toxicity and side effects.

The molecular targeted drugs for breast cancer treatment include: (1) trastuzumab (Herceptin) [45], pertuzumab (Perjeta) [46], T-DM1 [47], lapatinib [48], etc. The most representative antiHER-2 drug is trastuzumab. Trastuzumab is a recombinant DNA-derived humanized monoclonal antibody that selectively acts on the periphery of HER-2 cells, affecting the transmission of signals into the cell. It promotes the internalization of HER-2 receptor proteins and downregulates the activity of vascular endothelial growth factor (VEGF) [49]. It can also gather immune cells to kill tumor cells through antibody-dependent cell-mediated cytotoxicity (ADCC) [50]. Pertuzumab (trade name: Perjeta [51]) is a new HER-2 targeting monoclonal antibody. Pertuzumab and trastuzumab have different Her-2 extracellular binding sites and the combination of two targeted drugs increases the biological basis of patient benefit. (2) The anti-angiogenic drug bevacizumab (Avastin [52]) is a recombinant humanized monoclonal antibody that blocks intracellular tyrosine by competitively binding to human vascular endothelial growth factor. The activation of amino acid kinase blocks downstream signals to promote angiogenesis. (3) Inhibitors of PI3K/ AKT/mTOR pathway [53] include: everolimus [54], BKM120 [55], pictilisib, taselisib, and alpelisib. Everolimus is the first mTOR inhibitor approved for the treatment of hormone receptor positive/ Her-2 negative breast cancer. (4) PARP inhibitors such as iniparib, veliparib, and olaparib for BRCA1/2 mutations are undergoing relevant clinical trials (to be verified) [56].

Cyclin-dependent kinase 4 and 6 (CDK4/6) inhibitors such as Palbociclib [57] and Ribociclib [58], can selectively inhibit CDK4/6, 
restore cell cycle control, and block tumor cell proliferation [59]. In 2015, the FDA approved Palbociclib combined with letrozole was used as the first-line treatment for postmenopausal HR+ and HER2advanced breast cancer. The existing clinical data show that as new molecular targeted agents, CDK4/6 inhibitors have advantageous characteristics for the treatment of breast cancer, but the efficacy of combined treatment with CDK4/6 inhibitors and chemotherapeutics needs to be further studied.

\section{Breast cancer clinical medication 5G era-era of prevention}

The current mobile communication era has entered the $5 \mathrm{G}$ era. With this, the $5 \mathrm{G}$ era of breast cancer clinical drugs need to be studied.

Cervical and breast cancers are fatal for female patients. Cervical cancer is the only cancer worldwide that can be prevented and treated early. Thus, it is expected to be completely eradicated. Its incidence is second only to breast cancer. Currently, HPV vaccines [60] can be used for the prevention and treatment of cervical cancer. The HPV vaccine plays an increasingly important role in the prevention of cervical cancer. Although there are numerous medicines for breast cancer, vaccines for its prevention have not been created. Fortunately, many clinical trials on breast cancer vaccines are already on their way. Breast cancer vaccine therapy refers to the process of injecting the constructed tumor vaccine into the body to activate an immune response that produces specific and permanent immunity to tumor antigens. To develop an effective breast cancer vaccine, we must first select a highly specific tumor antigen as the target, select a suitable and efficient antigen presentation carrier, and thereafter overcome the tumor's immune tolerance mechanism and eliminate immunosuppression in the tumor microenvironment path. Currently, antigen presentation is mainly carried out using four methods: dendritic cells (DCs), whole tumor cells, polypeptide-related antigens, and viral plasmids. Dendritic cells are the most powerful antigen-presenting cells that are widely present in peripheral tissues. The whole tumor cell vaccine contains all the tumor antigens. It is one of the earliest and simplest tumor vaccines. It can theoretically produce a polyclonal immune response. However, because of its weak ability to activate immunity and easy formation of immune tolerance, immune adjuvant is required.
Breast cancer vaccines mainly include DC vaccines and specific antigen vaccines [61]. The tumor antigens used in the latter are mainly HER-2 and MUC-1 [62]. At the same time, human telomerase reverse transcriptase, survivin, and tumor proteins P53 and mammoglobulin A were also reported. The HER family regulates the growth and development of the normal breast tissue. The overexpression of HER-2 is related to breast cancer. The most used HER-2 polypeptide vaccines are E75 polypeptide, GP2 polypeptide, and AE37 polypeptide. NeuVaxTM [63] is a breast cancer HER-2 protein vaccine that can stimulate CD8+ cytotoxic $\mathrm{T}$ lymphocytes and CD8+ memory cells. It activates HER-2 protein-related immunogenic peptides and major histocompatibility complex class I antigen table bit. Phase II clinical trials have shown that NeuVaxTM can improve the overall survival rate of patients with HER-2 positive breast cancer, and it is well tolerated with mild adverse reactions. NeuVaxTM is the only breast cancer vaccine currently undergoing phase III clinical trials. MUC-1 is a high molecular weight glycoprotein, and the gene is located on chromosome lq21. Studies show that the quantity and quality of MUC-1 expression in tumor cells and normal cells are different. Approximately $90 \%$ of breast cancers have MUC-1 overexpression, so it is generally believed that MUC-1 is a tumor-associated antigen. Whether this can be successfully marketed depends on the hard work of scientific researchers.

\section{Conclusion}

Everything in the human world follows the principle of relativity. The only constant phenomenon is change. The change in time is unavoidable. It can allow human beings to progress into a new generation of civilization and intelligent society. Birth, old age, sickness, and death are the journeys that everyone must go through. Current living habits, state, and social environment may lead to the occurrence of human cancer. But diseases like cancer, if prevented, will bring breakthroughs to society. The changes in the country accelerated the ranks of social progress. The rapid progress of medicine and immunology provided new perspectives and approaches for tumor treatment. Similar to the development of HPV cervical cancer vaccines, three valence types have been developed since its launch. For women of different ages, the nine-valent vaccines can prevent more than $90 \%$ of the occurrence of cervical cancer. This is a crucial medicine for women. Physiologically, it can improve 
women's sense of security and greatly reduce the risk of suffering from cancer. The results of clinical trials show that breast cancer therapeutic and preventive vaccines made encouraging progress. However, the safety, effectiveness, and reliability of breast cancer vaccines need further investigation. Studies pointed out that in certain specific sequences, tumor vaccines combined with certain chemotherapeutic or targeted drugs (such as anthracyclines and cyclophosphamide drugs) can enhance the immune activity of the vaccine and improve the treatment effects. Researchers from various countries should work in coordination to explore and innovate, and develop cost-effective, safe, efficient, and practical breast cancer vaccines in the coming years.

If breast cancer vaccine is successfully developed, it will become an epoch-making news to prevent women with the highest incidence of cancer, and it increases the possibilities to improve their quality of life. Let us look forward to the arrival of the breast cancer cervical vaccine and the $5 \mathrm{G}$ era of breast cancer, bringing different choices and experiences to women.

\section{Conflict of Interest}

There has no conflict of interest.

\section{Author Contribution}

All authors contributed to the study conception and design. Material preparation, data collection and analysis were performed by Nan Zhang, Yan Zhang and Deqiao Wu. The first draft of the manuscript was written by Yan Zhang and all authors commented on previous versions of the manuscript. All authors read and approved the final manuscript.

\section{Bibliography}

1. Thani I and T Kasbe. "Breast Cancer: State -of- the-art causes and diagnosis". in 2nd International Conference on Data, Engineering and Applications (IDEA) (2020).

2. Fang J., et al. "Clinical Analysis of 67 Cases of Mastitis in Nonlactating Period". China Health Standard Management (2017).

3. Tavani A., et al. "Attributable risks for breast cancer in Italy: Education, family history and reproductive and hormonal factors". International Journal of Cancer 70.2 (2015): 159-163.
4. Anderson JAD. "Occupation as a modifying factor in the diagnosis and treatment of rheumatic diseases". Current Medical Research and Opinion 2.9 (1974): 521-528.

5. Yang J., et al. "The Relationship between Level of Estrogen in Postmenopausal Metastatic Breast Cancer and the Efficacy of Fulvestrant". Pharmaceutical Journal of Chinese Peoples Liberation Army (2016).

6. Braga., et al. "Cigarette smoking and the risk of breast cancer". American Journal of Epidemiology 131.2 (2016): 244-253.

7. Lidija RM and R Sinia. "Breast cancer susceptibility Gene 2 BRCA2”. Archive of Oncology 9.2 (2001): 115-118.

8. Turner N., et al. "Continued value of adjuvant anthracyclines as treatment for early breast cancer". Lancet Oncology 16.7 (2015): e362-e369.

9. Federico., et al. "New developments in antitumor anthracyclines". Pharmacology and Therapeutics (1997).

10. Dezhenkova LG., et al. "Topoisomerase I and II inhibitors: Chemical structure, mechanisms of action and role in cancer chemotherapy". Russian Chemical Reviews 83.1 (2014): 82.

11. Berthiaume JM and KB Wallace. "Persistent Alterations to the Gene Expression Profile of the Heart Subsequent to Chronic Doxorubicin Treatment". Cardiovascular Toxicology 7.3 (2007): 178-191.

12. Rodman MJ. "FDA approvals: '92 in review”. RN 56.3 (1993): 50-54.

13. Б СМ., et al. “Таксол в сочетании с доксорубицином при лечении больных диссеминированным раком молочной железы”. Вестникронцим.н.н:блохинарамн (1997).

14. Bouzada AO., et al. "Pharmaceutical formulation comprising taxane, a solid composition of taxane, a process for preparing said solid composition of taxane, a solubilizing composition of said solid composition of taxane, and a kit for the injectable formulation of taxane". US (2009). 
15. Sparreboom A., et al. "Cremophor EL-mediated alteration of paclitaxel distribution in human blood: clinical pharmacokinetic implications". Cancer Research 59.7 (1999): 1454-1457.

16. Kirikae T., et al. "Structural significance of the benzoyl group at the C-3'-N position of paclitaxel for nitric oxide and tumor necrosis factor production by murine macrophages". Biochemical and Biophysical Research Communications 245.3 (1998): 698-704.

17. Plosker GL and D Faulds. "Epirubicin. A review of its pharmacodynamic and harmacokinetic properties, and therapeutic use in cancer chemotherapy". Drugs 45.5 (1993): 788-856.

18. Gradishar and J William. "Albumin-bound paclitaxel: a next-generation taxane". Expert Opinion on Pharmacotherapy 7.8 (2016): 1041.

19. Meng S., et al. "Integrin-targeted paclitaxel nanoliposomes for tumor therapy". Medical Oncology 28.4 (2011): 1180-1187.

20. Oerlemans, C., et al. "Polymeric Micelles in Anticancer Therapy: Targeting, Imaging and Triggered Release". Pharmaceutical Research 27.12 (2010): 2569-2589

21. Schuyler D. "Metastatic Breast Cancer: Albumin-Bound Paclitaxel Found More Effective, Less Toxic than Standard Paclitaxel". Oncology Times 27.11 (2005): 1166-1177.

22. Nyman and W D. "Phase I and Pharmacokinetics Trial of ABI007, a Novel Nanoparticle Formulation of Paclitaxel in Patients With Advanced Nonhematologic Malignancies". Journal of Clinical Oncology Official Journal of the American Society of Clinical Oncology 23.31 (2005): 7785-7793.

23. Dhanikula AB., et al. "Pharmacokinetic study of paclitaxel as a 3-hour infusion in anIndian population: $135 \mathrm{mg} / \mathrm{m} 2$ vs. 175 mg/m2". Methods and Findings in Experimental and Clinical Pharmacology 23.2 (2001): 93-98.

24. Kawamura, M., et al. "Administration of low-dose albumin-bound Paclitaxel for thetreatment of advanced and recurrent breast cancer patients". Gan to Kagaku Ryoho Cancer and Chemotherapy 40.7 (2013): 909-912.
25. G., et al. "Enzyme entrapment in liposomes". Febs Letters (1971).

26. Liu D., et al. "Role of liposome size and RES blockade in controlling biodistribution and tumor uptake of GM1-containing liposomes". Biochimica Et Biophysica Acta 1104.1 (1992): 95.

27. Chee., et al. "Meta-analysis of clinical and preclinical studies comparing the anticancer efficacy of liposomal versus conventional non-liposomal doxorubicin". Journal of Controlled Release Official Journal of the Controlled Release Society 232 (2016): 255-264.

28. Zhang, Y., et al. "Construction and antitumor effects of antitumor micelles with cyclic RGD-modified anlotinib". Nanomedicine 28 (2020): 102224.

29. Gucalp, A and TA Traina. "Androgen receptor-positive, triplenegative breast cancer". Cancer 123.10 (2017).

30. Gillian., et al. "Letrozole”. American Journal of Cancer (2002).

31. Smedira HJ. "Practical issues in counseling healthy women about their breast cancer risk and use of tamoxifen citrate". Archives of Internal Medicine 160.20 (2000): 3034-3042.

32. Yongzhen H., et al. "Effect of endogenous glucocorticoid on expression of p53 and Bcl-2 proteins in osteocyte apoptosis in avascular necrosis of the femoral head induced by alcoholism". Guizhou Medical Journal (2004).

33. Wiseman LR and KL Goa Formestane. "A review of its pharmacological properties and clinical efficacy in the treatment of postmenopausal breast cancer". Drugs Aging 9.4 (1996): 292306.

34. Mouridsen H., et al. "Letrozole therapy alone or in sequence with tamoxifen in women with breast cancer". Breast Diseases A Year Book Quarterly 21.2 (2009): 168-169.

35. Carraway $\mathrm{H}$ and AC Wolff. "Anastrozole is safer and may be more effective than tamoxifen in postmenopausal women with early-stage breast cancer". Cancer Treatment Reviews 30.3 (2004): 303-307. 
36. Goss PE., et al. "Exemestane for Breast-Cancer Prevention in Postmenopausal Women". New England Journal of Medicine 364.25 (2011): 2381-2391.

37. a, D.A.M.C.M., et al. "Bone density and structure in healthy postmenopausal women treated with exemestane for the primary prevention of breast cancer: a nested substudy of the MAP.3 randomised controlled trial" (2012).

38. Gampe RT., et al. "Structural basis for autorepression of retinoid $\mathrm{X}$ receptor by tetramer formation and the AF- 2 helix". Genes and Development 14.17 (2000): 2229.

39. Stenbygaard LE., et al. "Toremifene and tamoxifen in advanced breast cancer - a double-blind cross-over trial". Breast Cancer Research and Treatment 25.1 (1993): 57-63.

40. Walsh and W Brian. "Effects of Raloxifene on Serum Lipids and Coagulation Factors in Healthy Postmenopausal Women". Jama 279.18 (1998): 1445-1451.

41. Anthony., et al. "Fulvestrant versus anastrozole for the treatment of advanced breast carcinoma". Cancer 104.2 (2005).

42. István P. "Role of gonadotropin-releasing hormone (GnRH, LH-RH) analogues in the therapy of cancer". Magyar Onkológia 43.1 (1999): 19-24.

43. Navarro MA., et al. "[The estrogen receptor/progesterone receptor quotient as an index of the transcriptional activity of estrogen receptors in breast cancer]". Medicina Clní ica 104.18 (1995): 681.

44. Lei F., et al. "Targeted therapy in breast cancer: what's new?" Swiss Medical Weekly141 (2011): w13231.

45. Andre F., et al. "Everolimus, Paclitaxel, Trastuzumab, Breast Cancer". Journal of Clinical Oncology Official Journal of the American Society of Clinical Oncology 28.34 (2010): 51105115.

46. Portera CC., et al. "Cardiotoxicity of trastuzumab, Cardiac toxicity, pertuzumab, breast cancer". Clinical Cancer Research An Official Journal of the American Association for Cancer Research 14.9 (2008): 2710.
47. Gabriel., et al. "HER2 Directed Antibody-Drug-Conjugates beyond T-DM1 in Breast Cancer". International Journal of Molecular Sciences 20.5 (2019): 1115.

48. Bilancia D., et al. "Lapatinib, breast cancer". Annals of Oncology 6 (2007): vi26-30.

49. Burstein HJ., et al. "VEGF, Breast Cancer, Bevacizumab, Vinorelbine, Marker". Clinical Cancer Research An Official Journal of the American Association for Cancer Research 14.23 (2008): 7871-7877.

50. Juranic Z., et al. "The importance of antibody dependent cellmediated cytotoxicity (ADCC) for breast cancer response to trastuzumab - Herceptin". Archive of Oncology 10.3 (2002): 162-163.

51. Release FN. "FDA Approves Perjeta for Neoadjuvant Breast Cancer Treatment". Molecular and Cellular Pharmacology (2013).

52. Reports, F.S. "FDA begins process to remove breast cancer indication from Avastin label” (2010).

53. Li D., et al. "Tumor associated macrophages secrete CCL2 and induce tamoxifen resistance by activating PI3K/Akt/mTOR in breast cancer". Cancer Science 111.1 (2019).

54. Awada, A., et al. "mTOR, RAD001, everolimus, breast cancer". European Journal of Cancer 44.1 (2008): 84-91.

55. Hu Y., et al. "Effects of PI3K inhibitor NVP-BKM120 on overcoming drug resistance and eliminating cancer stem cells in human breast cancer cells". Cell Death and Disease 6.12 (2015): e2020.

56. Salazar R., et al. "BRCA1-2 mutations in breast cancer: Identification of nine new variants of BRCA1-2 genes in a population from central Western Spain". Cancer Letters 233.1 (2006): 172-177.

57. Seelbach-Gobel., et al. "Berlin, 22nd May 2017 252nd Opinion of the DGGG: Opinion on Palbociclib (Breast Cancer)". Geburtshilfe Und Frauenheilkunde (2017). 
58. Hortobagyi GN., et al. "Ribociclib as First-Line Therapy for HR-Positive, Advanced Breast Cancer". The New England Journal of Medicine 375.18 (2016): 1738-1748.

59. Alajez NM. "Abstract 301: Concurrent targeting of BMI1 and CDK4/6 inhibited breast cancer tumorigenicity in vitro and in vivo". in Proceedings: AACR Annual Meeting 2019 March 29-April 3, 2019 Atlanta, GA (2019).

60. Konno R. "HPV (human papillomavirus) vaccine”. Nihon Rinsho 4.7 (2012): 175-183.

61. Bella SD., et al. "Altered maturation of peripheral blood dendritic cells in patients with breast cancer". British Journal of Cancer 89.8 (2003): 1463.

62. Cleanthi., et al. "Immunohistochemical study of p185 HER2 and DF3 in primary breast cancer and correlation with CA-153 serum tumor marker". International Journal of Gynecological Cancer Official Journal of the International Gynecological Cancer Society 12.1 (2002): 74-79.

63. "RXi Pharmaceuticals Presents Additional Positive Phase 2 Efficacy Results for Combination of NeuVaxTM (E75) and Trastuzumab Breast Cancer at ASCO". Biomedical Market Newslet$\operatorname{ter}(2011)$.

\section{Assets from publication with us}

- Prompt Acknowledgement after receiving the article

- Thorough Double blinded peer review

- Rapid Publication

- Issue of Publication Certificate

- High visibility of your Published work

Website: www.actascientific.com/

Submit Article: www.actascientific.com/submission.php

Email us: editor@actascientific.com

Contact us: +919182824667

Citation: Zhenzhong Zhang, Xia Xu., et al. "Arrival of the 5G Era of Clinical Medicine for Breast Cancer”. Acta Scientific Pharmaceutical Sciences 5.3 (2021): $17-26$. 